\title{
UNA REVISIÓN DE LOS ESTILOS DE VIDA DE ESTUDIANTES UNIVERSITARIOS IBEROAMERICANOS ${ }^{1}$
}

\author{
A REVIEW OF LATIN AMERICAN UNIVERSITY \\ STUDENTS' LIFESTYLES
}

\author{
Elizabeth Magdalena Bastías Arriagada* \\ Jasna Stiepovich Bertoni ${ }^{* *}$
}

\begin{abstract}
RESUMEN
Durante los últimos años la sociedad ha experimentado profundos cambios en materia de salud. Estos cambios se deben a una serie de factores del entorno que gatillan el desarrollo de determinados estilos de vida, pudiendo ser éstos de riesgo o protectores para la salud de las personas. Objetivo: Indagar respecto al concepto de estilo de vida y los estilos de vida de estudiantes universitarios iberoamericanos según la evidencia disponible. Material y método: Se realizó una revisión bibliográfica, manual y electrónica de artículos vinculados a estilos de vida en población universitaria iberoamericana a partir del año 2002. Adicionalmente, se recabó información referida al concepto de estilos de vida y se recopilaron antecedentes estadísticos disponibles del Ministerio de Salud de Chile y de otras entidades que investigan estilos de vida en los jóvenes. Resultados: La mayor parte de los trabajos desarrollados han estudiado de manera descriptiva los estilos de vida. La alimentación y ejercicio, la conducta sexual, el consumo de tabaco, alcohol y drogas, son las prácticas más estudiadas. Conclusión: Los jóvenes universitarios son una población vulnerable que debe ser investigada de manera particular, para crear y evaluar programas de intervención acorde a sus necesidades.
\end{abstract}

Palabras clave: Estilo de vida, factores de riesgo, estudiantes, universidades.

\begin{abstract}
During the last years, our society has experienced profound changes on health matters. These changes are due to a number of environmental factors that trigger the development of certain lifestyles which could either endanger or protect people's health. Objective: To research about the concept of lifestyle and Iberoamerican university students' lifestyles considering the available evidence. Methods: A manual and electronic bibliographic review of articles concerning lifestyles in a Iberoamerican university population from the year 2002, was done. In addition, information about the concept of lifestyles and statistical data available from the Ministry of Health in Chile and other research organizations about young people's lifestyles, were collected. Results: Most of the researches carried out, have studied lifestyles in a descriptive form. Nutrition and physical exercising, sexual behavior, tobacco, alcohol and drug consumption are the most studied habits. Conclusions: Young university students are a vulnerable population that must be studied in a particular way in order to create and assess intervention programs according to their needs.
\end{abstract}

Key words: Lifestyle, risk factors, students, universities.

Fecha recepción: 15/04/13. Fecha aceptación: 19/07/14.

\footnotetext{
${ }^{1}$ Manuscrito derivado de tesis de Doctorado en Enfermería de la Universidad de Concepción, titulado: "Estilo de vida en universitarios y factores psicosociales".

*Enfermera. Docente Depto. Enfermería. Universidad de Concepción, Concepción. E-mail: elibastias@udec.cl.

${ }^{* *}$ Enfermera. Docente Depto. Enfermería. Universidad de Concepción, Concepción. E-mail: jstiepov@udec.cl.
} 


\section{INTRODUCCIÓN}

Los avances científicos han propiciado que la sociedad experimente cambios importantes en los indicadores vinculados a salud. Si bien éstos han permitido un incremento en la esperanza de vida al nacer de la población mundial, también han significado la aparición de problemas de salud emergentes que pudiesen ser más difíciles de erradicar que los que afectaban antaño, pues su raíz radica en los estilos de vida de las personas.

El Informe sobre la Salud en el Mundo 2002 (1) señala que de un conjunto de 25 grandes riesgos evitables seleccionados y estudiados en detalle, entre los más importantes a nivel mundial se encuentran los siguientes: prácticas sexuales sin protección, presión arterial elevada, tabaco, alcohol, niveles de colesterol elevados, y sobrepeso/obesidad, todos vinculados a estilos de vida. En su conjunto, estos riesgos representan un tercio de los años de vida sana perdidos en todo el mundo. Los principales riesgos examinados en el informe dan lugar a importantes reducciones de la esperanza de vida sana: en promedio, unos cinco años en los países en desarrollo y diez años en los países desarrollados. El número de años de vida sana perdidos a causa de esos riesgos varía según las regiones; es así como en la mayor parte de América Latina se calcula esta pérdida en 6,9 años (1).

Chile, por su parte, ha experimentado una rápida transición epidemiológica entre los años '70 y '90: “De acuerdo a estos cambios, se comienza a trabajar en nuevas orientaciones de política y salud, realizándose en 1995 el Estudio de Carga de Enfermedad que demostró, que el 75\% de los Años de Vida Saludable (AVISA) perdidos en Chile se debían a Enfermedades Crónicas No Transmisibles (ECNT) y a problemas de salud mental" (2). Esto dio origen a cambios en el enfoque de prioridad entonces destinados al área materno-infantil y a enfermedades infecciosas.

Salinas y Vio del Río (3) señalan en una publicación que según el censo de 2002, Chile es un país altamente urbanizado $(86 \%$ de población urbana), habiendo mejorado el acceso al agua potable, alcantarillado, educación y servicios de salud. Sin embargo, aparecen problemas relacionados con el deterioro de la calidad de vida, especialmente en los más pobres, como son la alimentación inadecuada, el sedentarismo, el consumo excesivo de tabaco, alcohol y drogas, la contaminación del aire y falta de espacios para recreación en las grandes ciudades. Si bien Chile aumentó al doble su ingreso per cápita en la década del 90, gran parte del aumento de ingreso, en especial en el sector más pobre, se destinó a comida rica en grasa y azúcar refinada, se disminuyó la actividad física y se incrementó el consumo de tabaco y alcohol, que inciden directamente en la salud de las personas. Esto significó un aumento explosivo de la obesidad y sedentarismo, así como del tabaquismo en la población chilena, especialmente en los niños, adolescentes y mujeres en edad fértil; fenómeno que también se ha ido produciendo en muchos países.

Datos de la última Encuesta Nacional de Salud (ENS) (4) muestran un significativo aumento de las condiciones de riesgo para la salud respecto de la medición anterior (ENS 2003). En relación a sobrepeso y obesidad se observó incremento de 37,8 a 39,3\% y en obesidad de 23,2 a 25,1\%. En ambos casos existen diferencias por sexo, siendo mayor en los hombres en condición de sobrepeso y mayor en el caso de las mujeres en condición de obesidad. En el caso de consumo de tabaco, quienes se consideran fumadores diarios a nivel nacional responden afirmativamente $40,6 \%$ de los encuestados, siendo menor el porcentaje en mujeres, y mayor el problema en la población que señala tener estudios $>12$ años (50,3\%). Respecto del consumo de alcohol, la respuesta varía si la pregunta es formulada como "Bebedor diario y moderado" (al menos 5 días en la última semana) $(1,9 \%)$, o si se aplica la escala de dependencia alcohólica AUDIT (10,9\%). Respecto al uso 
de preservativo los varones refieren su uso en $57 \%$ (mujeres 50,5\%). Al ser consultados por su participación social $39,1 \%$ de los hombres refiere pertenecer a un grupo $(28,8 \%$ de las mujeres).

La Sexta Encuesta Nacional de la Juventud (5) señala que la población joven entre 15 a 19 años era de $1.483 .285(35,4 \%)$, mientras que el tramo etario de 20 a 24 años era de 1.433.402 jóvenes $(34,2 \%)$. Se concentran mayoritariamente en la Región Metropolitana (40,8\%) (Región del Bío-Bío (12\%) y de Valparaíso (10,2\%)). Cursan Educación Universitaria 23,2\% de este grupo (970.798). Alrededor de un $8 \%$ del grupo total trabaja y estudia. La gran mayoría de las y los jóvenes chilenos $(91,1 \%)$ pertenece a algún tipo de sistema de previsión de salud (Fondo Nacional de Salud -FONASA- 73,3\%, Instituciones de Salud Previsional-ISAPRE-14\%). Las obligaciones económicas con la familia son referidas como razón fundamental para buscar trabajo (11\% para ayudar a sus padres y $11 \%$ para el financiamiento de sus estudios), lo que se acentúa en los estratos económicos más bajos y en los varones. En cuanto a la participación juvenil en una organización, los varones son más proclives (club deportivo 38,2\%, similar a la ENS 2009-2010), siendo mayor la participación en el grupo etario que va de los 15 a 19 años. Dentro de las razones por las que estarían dispuestos a formar parte de un grupo, 52,9\% lo haría si tuviera más tiempo libre y $47 \%$ si hubiera alguna organización que satisfaga sus intereses. Respecto a la confianza en las instituciones 38,8\% tiene alta confianza en las Universidades, $62,5 \%$ se identifica o pertenece a alguna religión, en tanto que $45,4 \%$ señala que nunca asisten a la iglesia. Son sexualmente activos $75,7 \%$ y la edad de inicio de relaciones sexuales promedio de la muestra fue 16,7 años (en varones 16,4 años y en mujeres 17,1 años). Cuando son consultados respecto al tipo de parejas durante los últimos doce meses, la mayoría refiere relaciones heterosexuales (hombres 94,5 y $97,6 \%$ de las mujeres), las relaciones homosexuales en hombres es de $4,7 \%$ y en mujeres $1,7 \%$. El método utilizado en la última relación sexual tanto para condón preservativo y píldora anticonceptiva fue de $44,2 \%$, siendo mayor el uso de preservativo en varones $(55,9 \%)$ y en el grupo de 15 a 19 años $(65,1 \%)$. La píldora anticonceptiva es más usada en mujeres $(46,8 \%)$ y su uso se incrementa con la edad. Las razones para el uso de condón: 90,6\% para prevenir un embarazo; $39,7 \%$ para proteger de VIH/SIDA y $38,3 \%$ para proteger de otras infecciones de transmisión sexual. Quienes no utilizan métodos de anticoncepción y/o prevención señalan que fue por irresponsabilidad $(23,4 \%)$, no le gusta usar ninguno de los métodos que conoce $(15,3 \%)$, quería quedar embarazada $(10,6 \%)$, no pudo conseguir un método anticonceptivo $(9,6 \%)$, su pareja no quería usar ningún método $(9,1 \%)$. Respecto al consumo de drogas, el 59,2\% señala que consume drogas lícitas, $22 \%$ no consume drogas, $18,5 \%$ consume drogas lícitas e ilícitas. Respecto al consumo de alcohol es referido por $82,5 \%$ de los universitarios, $76,7 \%$ del grupo de jóvenes entre 20 y 24 años.

Lo anterior da cuenta de una clara transición epidemiológica tanto a nivel mundial como local, dando origen a diversas iniciativas relacionadas con la Promoción de Salud en Chile a partir del año 1972. Entre estos documentos cabe destacar Guías para Universidades Saludables, publicado el año 2006 por el Ministerio de Salud (MINSAL) (6), documento que hace un relevamiento para Chile de las experiencias nacionales e internacionales de las vivencias entre las universidades y la promoción de la salud, así como aspectos organizativos y estratégicos de una universidad saludable.

El Gobierno de Chile, a través del Ministerio de Salud, se ha hecho parte de esta sentida necesidad de cuidar a la población de adolescentes y jóvenes, entre los que se cuentan los universitarios, a través del "Programa de Salud Integral de Adolescentes y Jóvenes", que acoge a adolescentes entre 10 y 19 años 
y jóvenes entre 20 y 24 años, creado según Decreto $N^{\circ} 132$ de diciembre de 2010 y "tiene el propósito de mejorar el acceso y la oferta de servicios, diferenciados, integrados e integrales, en los distintos niveles de atención del sistema de salud, articulados entre sí, que respondan a las necesidades de salud actuales de adolescentes y jóvenes, con enfoque de género y pertinencia cultural, en el ámbito de la promoción, prevención, tratamiento y rehabilitación, incorporando a sus familias y la comunidad" (7), La iniciativa nace debido a que, de acuerdo a los indicadores disponibles, los hombres mueren antes y se enferman más que las mujeres, lo que además de variables culturales y de género podría deberse a falta de oferta específica de prestaciones y servicios para ellos (8).

Los antecedentes anteriormente expuestos cobran importancia en la población universitaria, que ve modificados muchos de sus comportamientos durante un período promedio de al menos 5 años y que en concomitancia transitan desde la adolescencia a la adultez joven, iniciando la toma de decisiones en base a las situaciones de vida que se les van presentando, muchas de ellas estarán basadas en el deseo de ser aceptados por sus pares o simplemente por socialización (9). Algunos autores señalan que "esta situación de cambio puede ser un evento estresante difícil de sobrellevar sobre todo para aquellos que vivirán fuera del hogar durante este período" (10).

Objetivo: Indagar respecto al concepto de estilo de vida y los estilos de vida de estudiantes universitarios iberoamericanos según la evidencia disponible.

\section{MATERIAL Y MÉTODOS}

La revisión fue efectuada mediante búsqueda manual y electrónica de artículos relacionados con estilos de vida como concepto, considerando con especial énfasis aquéllos relacionados con estudiantes universitarios iberoamericanos, en fuentes de indexación ISI, LILACS, SCIELO, MEDLINE. Los términos de búsqueda fueron: estilo de vida, factores de riesgo, estudiantes, universidades. La selección de los artículos originales se efectúo además considerando un período de al menos 5 años para artículos relacionados con experiencias investigativas y para los aspectos teórico-conceptuales el período se extendió al menos a 10 años. Se analizaron los resultados y los antecedentes se incorporaron en una base de datos a fin de contrastar similitudes y diferencias para la organización del presente artículo.

\section{RESULTADOS Y DISCUSIÓN}

\section{Estilos de Vida}

Los estilos de vida como concepto han sido desarrollados desde hace algunas décadas por la sociología y la psicología; sin embargo, en el ámbito de la salud fue Lalonde (11) quien lo explica dentro de un modelo de salud, compuesto por cuatro elementos: la biología humana, el medio ambiente, los estilos de vida y la organización de los servicios de salud. Los estilos de vida consisten en la gama de decisiones que el individuo toma y que le afecta, sobre las que tiene mayor o menor control; si éstas son malas decisiones desde el punto de vista de salud, el individuo crearía un riesgo autoimpuesto, que le puede predisponer a enfermar o incluso llevar a la muerte, reconociéndose la multivariabilidad en la toma de decisiones (11). El constructo estilo de vida se asocia a las ideas de comportamiento individual y patrones de conducta. Según lo mencionan Guerrero et al. (12), hacen referencia a la manera de vivir, a una serie de actividades, rutinas cotidianas o hábitos. Al conjunto de estas prácticas se les denomina hábitos o comportamientos sostenidos, existiendo los que mantienen la salud y pro- 
mueven la longevidad (factores protectores) y aquellos que la limitan o resultan dañinos y reducen la esperanza de vida (conductas de riesgo) (13).

En la trigésimo primera sesión del comité regional de la OMS para Europa, se presentó una definición del estilo de vida, considerándola: "Una forma general de vida basada en la interacción entre las condiciones de vida en un sentido amplio y los patrones individuales de conducta determinados por factores socioculturales y características personales" (14). No se introdujo una definición concreta para el término de estilo de vida saludable, por lo que la mayoría de los autores lo consideran como "un conjunto de patrones conductuales o hábitos que guardan una estrecha relación con la salud de las personas" (15). Como señalan De la Torre y Tejada "los estilos de vida son un modo de concebir la realidad y proyectarse en la vida" (16). En general, se entiende el estilo de vida como la forma de vida de las personas o de los grupos (17). En lo que no todos coinciden es si estos patrones conductuales son elegidos voluntaria o involuntariamente por los individuos (17). Sin embargo, la orientación psicosocial entiende la elección como involuntaria en cierta medida, reconociendo la influencia de las variables psicosociales en la adquisición y mantenimiento del estilo de vida (18), toda vez que se aprenden a lo largo del proceso de socialización del individuo y una vez adquiridos son difíciles de modificar (19).

\section{Estilos de vida en la población universitaria}

Palacio et al. encontraron en universitarios colombianos conductas de riesgo como el sedentarismo, malos hábitos de alimentación e incluso han considerado el suicidio algunos de ellos (20). Este estudio arroja similares resultados a uno realizado en la Universidad de Chile, Santiago de Chile (Agronomía, Ingeniería en Recursos Naturales Renovables, Ingeniería Forestal e Ingeniería en Madera) en el que se comparan alumnos de primer año
(PA) versus alumnos de tercer año (TA) de permanencia, en donde se buscaba evaluar a ambos grupos en base a su calidad de vida y estado nutricional referidos. Los alumnos de primer año presentaron mejor percepción en su vida sexual, de pareja y bienestar, menor consumo de alcohol y estado nutricional adecuado en relación con el grupo de tercer año. El método anticonceptivo más utilizado fue el anticonceptivo oral (bajo porcentaje usa el condón) (21). Por su parte, Trujillo-Hernández et al. realizaron un estudio en una universidad mexicana para determinar la frecuencia y factores de riesgo para sobrepeso y obesidad en jóvenes universitarios. El $31,6 \%$ de la muestra presentó sobrepeso y obesidad mayor en hombres que en mujeres (22).

Alonso et al. (23) realizaron un estudio para determinar los comportamientos de riesgo para la salud en estudiantes colombianos recién ingresados a una universidad privada: el $13,5 \%$ presentó sobrepeso y 2,6\% obesos y 1 de 5 camina todos los días menos de media hora. Para controlar el estrés: 43,3\% escucha música; el $67.2 \%$ ha consumido alcohol; 35,2\% ha fumado. El 51,5\% inició vida sexual; $56,6 \%$ utiliza método para planificación familiar. Prevalencia de enfermedades de transmisión sexual: 1.1\%. En el último año el 4,3\% ha considerado el suicidio; de éstos $65,2 \%$ han realizado intentos. Concluyen que se hace imprescindible el desarrollo de estrategias para modular la influencia de las conductas de riesgo sobre la salud a corto y largo plazo. Castillo y Molina-García (24) encontraron en un grupo de universitarios españoles, que el nivel de actividad física de los participantes era moderada; se percibían competentes, tenían una alta autoestima, se encontraban satisfechos con su vida y se sentían con alta vitalidad. La competencia física percibida se asoció positivamente con la autoestima, la satisfacción con la vida y vitalidad subjetiva. Concluyeron que el aumento de la actividad física además de contribuir a tener menos grasa corporal, 
aumenta el bienestar psicológico y mejora la autopercepción. Por su parte, Castillo et al. (25) realizaron un estudio en universitarios españoles, encontrando que la percepción de competencia deportiva favoreció el bienestar psicológico directamente, e indirectamente a través de la motivación autónoma; además, la percepción de competencia se asoció negativamente con el malestar psicológico. Concluyeron que sentirse competentes lleva a los sujetos a tener una percepción más favorable sobre sus vidas y a sentirse con más energía y viveza, lo que favorece su salud mental. Muñoz y Ruiz (26) evaluaron el nivel de autoestima de universitarios españoles y su posible relación con comportamientos de riesgo, los resultados refieren puntuación de autoestima mayoritariamente entre medio bajo y medio alto. No hay significación en la correlación entre comportamiento sexual y nivel de autoestima y, sorprendentemente, hubo mayor consumo de alcohol, cannabis, cocaína, drogas de diseño y anfetaminas en el grupo que refirió mayor autoestima que, señalan, pudiera deberse a que la alta autoestima se relaciona con la experimentación. Ramos et al. (27) investigaron estudiantes colombianos encontrando que el fitness de la comunidad estudiantil está por debajo de los valores esperados, con un elevado porcentaje de grasa; el perímetro de cintura estuvo entre alto y muy alto para el 5,2\% de los evaluados, el IMC mostró que un $7 \%$ está en peso bajo y el $21,6 \%$ en exceso. La escasa actividad física realizada por la comunidad estudiantil se refleja en bajo nivel de las capacidades condicionales y elevado porcentaje de grasa. Troncoso et al. (9) realizaron un estudio fenomenológico en el que encontraron que no existen diferencias entre carreras de diversas áreas y las de salud y otros estudios manifiestan de manera más preocupante que la población de personas que cursan carreras de salud presentan incluso conductas más lesivas, que las hace más proclives a la adquisición de ECNT.

Páez y castaño (28) exploraron algunos componentes de los estilos de vida de estudiantes de Medicina colombianos y encontraron que el mejor apoyo referido por los estudiantes para tener estilos de vida saludable es la familia y que no adhirieron a programas creados como red de apoyo dentro de la Institución Universitaria. Frente a la salud sexual y reproductiva, el consumo de tabaco-alcohol y hábitos alimenticios, no perciben el riesgo como cercano (sensación de invulnerabilidad). Se preguntan los autores si los factores de riesgo estarían relacionados con la alta exigencia académica que plantean las carreras de salud. Oviedo et al. (29) publican el 2006 un estudio de factores de riesgo de enfermedades crónicas no transmisibles (ECNT) en estudiantes de medicina venezolanos encontrando que los alumnos presentaron sedentarismo, hábitos alcohólicos, hábitos tabáquicos, consumo de alimentos nocivos, sobrepeso u obesidad. Montero et al. (30) evaluaron hábitos de alimentación y otros estilos de vida de acuerdo a los conocimientos sobre nutrición y dietética de universitarios de salud españoles que cursaban dicha asignatura (Enfermería, Farmacia, Nutrición y Dietética y Podología), encontraron que $67 \%$ estaba en el rango normopeso, sólo $40 \%$ de los encuestados realizaba ejercicio de manera regular. Los autores concluyeron que la población no ha modificado sensiblemente sus hábitos pese a contar con la información suficiente, estando sus decisiones limitadas por factores de socialización como del entorno. Sanabria-Ferrand et al. (31) indagaron en profesionales de salud colombianos para establecer la relación entre su formación y su estilo de vida. Los resultados revelaron que tanto los profesionales médicos como enfermeras(os) tienen comportamientos poco saludables o nada saludables: presentan sobrepeso u obesidad, poca o nula actividad física, bajo porcentaje tiene buena calidad en su alimentación. Los autores señalan que es necesario desarrollar estudios que midan determinantes biológicos, psicológicos, sociales y culturales de los estilos de vida. 


\section{CONCLUSIÓN}

Los hallazgos señalados muestran una población vulnerable que en algunos aspectos presenta mayores problemas que la población general, situación preocupante si consideramos que en este grupo existen personas que poseen los conocimientos necesarios para llevar a cabo adecuadas conductas pero que, aparentemente, no perciben el riesgo, siendo quienes menos conductas saludables realizan.

Los estilos de vida son conductas que involucran decisión personal, sea voluntaria o involuntaria. La mayor parte de la evidencia disponible es descriptiva, siendo la alimentación y ejercicio, la conducta sexual, el consumo de tabaco, alcohol y drogas, los estilos más estudiados. No se debe olvidar que existen otros estilos como el ocio, la recreación, el sueño y reposo que deben ser incorporados en futuras investigaciones. Los registros porcentuales de las distintas conductas ligadas a estilos varían en distintos países y dentro de Chile, sin embargo, su aparición es una constante en todos los grupos. Así el registro de sobrepeso y obesidad es mayor en hombres que en mujeres, especialmente en Brasil (50\%), considerando la variable sexo y cuando se considera todo el grupo es mayor en algunas regiones de España, Colombia y Chile.

Respecto a la actividad física llama la atención la poca adhesión a realizarla por parte de los universitarios, registrándose alta prevalencia de sedentarismo en República Dominicana y en algunas ciudades chilenas. El consumo de alcohol y tabaco es alto y de similares proporciones en los grupos estudiados, mientras que el consumo de drogas, si bien es un bajo porcentaje, la situación es diferente en algunos estudios colombianos, que reportan más alto consumo que estudiantes chilenos o españoles.

Pese a los esfuerzos que en materia de salud se están realizando, a los que se suman las instituciones universitarias a través de la red de Universidades Promotoras en Salud, los autores coinciden en la necesidad de realizar estudios en los que se indague respecto a factores físicos, sociales y psicológicos que pudiesen influir sobre los estilos de vida y con los resultados generar propuestas de intervención que fomenten estos estilos, considerando las características y el contexto en que se desenvuelve cada grupo estudiado, aspecto que se estima relevante para el logro de conductas saludables en poblaciones específicas.

\section{REFERENCIAS}

1. Organización Mundial de la Salud. Informe sobre la salud en el mundo 2002. [Internet] Ginebra: OMS: 2002 [citado 24 de junio 2012] 290 p. Disponible en: http:// www.who.int/whr/2002/es/index.html

2. Crovetto M, Vio del Río F. Antecedentes internacionales y nacionales de la promoción de salud en Chile: lecciones aprendidas y proyecciones futuras. Rev Chil Nutr. 2009; 36(1): 32-45.

3. Salinas J, Vio del Río F. Promoción de la Salud en Chile. Rev Chil Nutr. 2002; 29 (suppl.1): 164-173.

4. Ministerio de Salud, Gobierno de Chile. Encuesta Nacional de Salud 2009-2010 [Internet]. Santiago, Chile: MINSAL; 2009 [citado 17 agosto 2012] Disponible en: http://web.minsal.cl

5. Instituto Nacional de la Juventud. Sexta Encuesta Nacional de la Juventud [Internet]. Santiago, Chile: INJUV; 2009 [citado 1 agosto 2012]. Disponible en: http:// www.injuv.gob.cl

6. Ministerio de Salud de Chile. Guía para Universidades Saludables y otras instituciones de Educación Superior. [Internet]. Santiago, Chile: MINSAL; 2006 [citado 07 julio 2012] Disponible en: http://www. inta.cl/materialEducativo/guia_universidades_saludables2006.pdf

7. Ministerio de Salud de Chile. Programa 
de Salud Integral de Adolescentes y Jóvenes [Internet]. Santiago, Chile: MINSAL; 2012 [citado 1 agosto 2012]. Disponible en: http://www.minsal.gob.cl/portal/url/ page/minsalcl/g_proteccion/g_psiayj/ presentacion_psiayj.html

8. Ministerio de Salud de Chile. Situación actual de la salud del hombre en Chile [Internet]. Santiago, Chile: MINSAL; 2011 [citado 1 agosto 2012]. Disponible en: http://www.minsal.gob.cl/portal/url/item/ b7e8f68be82d7f2fe040010165013351.pdf

9. Troncoso C, Amaya JP. Factores sociales en las conductas alimentarias de estudiantes universitarios. Rev Chil Nutr. 2009; 36(4): 1090-1097.

10. Agudelo-Vélez D, Casadiegos-Garzón C, Sánchez-Ortíz D. Relación entre esquemas maladaptativos tempranos y a características de ansiedad y depresión en estudiantes universitarios. Univ. Psychol. 2009; 8(1): 87-104.

11. Lalonde M. A New Perspective on the Health of Canadians: A Working Document Ministry of Supply and Services Canada [Internet]. Ottawa: Health Canada; 1981 [citado 17 junio 2012] Disponible en: http://www.hc-sc.gc.ca

12. Guerrero Montoya L, León Salazar A. Estilo de vida y salud: un problema socioeducativo, antecedentes. Educere. 2010; 14 (49): 287-296.

13. Jenkins D. Mejoremos la salud a todas las edades. Un manual para el cambio de comportamiento [Internet]. Washington, D.C: Organización Panamericana de la Salud; 2005 [citado 17 agosto 2012] 448 p. Disponible en: http://iris.paho.org

14. Kickbusch I. Life-styles and health. Soc Sci Med. 1986; 22(2): 117-124.

15. Pastor Y. Un estudio de la influencia del autoconcepto multidimensional sobre el estilo de vida saludable en la adolescencia media. [Tesis doctoral]. [Valencia, España]: Universitat de València; 1999.

16. Torre de la S, Tejada J. Estilos de Vida y
Aprendizaje Universitario. Revista Iberoamericana de Educación [Internet]. 2007 [citado 25 mayo 2011]; 44: 101-131. Disponible en: http://www.rieoei.org/ rie44a06.pdf

17. Pastor Y, Balaguer I, García-Merita ML. Estilo de vida saludable en la adolescencia media: análisis diferencial por curso y sexo. Revista de Psicología de la Salud, [Internet]. 2000 [citado 31 mayo 2012]; 12(2). Disponible en: http://www.psicothema.com/pdf/3170.pdf

18. Barrios-Cisnero H. Estilo de Vida Saludable y Espiritualidad. Monografía s/p. Mérida, Venezuela; 2007.

19. Pastor Y, Balaguer I, García-Merita M. Una revisión sobre las variables de estilos de vida saludables. 1998. Rev de Psicol de la salud; 10(1): 15-52.

20. Palacio A, Pérez LM, Alcalá MÁ, Lubo G, Gálvez A, Consuegra A. Comportamientos de riesgo para la salud en estudiantes colombianos recién ingresados a una universidad privada en Barranquilla (Colombia). Salud, Barranquilla. 2008; 24(2): 235-247.

21. Durán S, Castillo M, Vio del R F. Diferencias en la calidad de vida de estudiantes universitarios de diferente año de ingreso del Campus Antumapu. Rev Chil Nutr. 2009; 36(3): 200-209.

22. Trujillo-Hernández B, Vásquez C, Almanza-Silva JR, Jaramillo-Virgen ME, Mellin-Landa TE, Valle-Figueroa OB et al. Frecuencia y factores de riesgo asociados a sobrepeso y obesidad en universitarios de Colima, México. Rev Salud Publica. $2010 ; 12(2)$ : 197-207.

23. Alonso LM, Pérez MÁ, Alcalá G, Lubo A, Consuegra A. Comportamientos de riesgo para la salud en estudiantes colombianos recién ingresados a una universidad privada en Barranquilla (Colombia). Salud, Barranquilla. 2008; 24(2): 235-247.

24. Castillo I, Molina-García J. Adiposidad corporal y bienestar psicológico: efectos 
de la actividad física en universitarios de Valencia, España. Rev Panam Salud Pública. 2009; 26(4): 334-40.

25. Castillo I, Molina-García J, Alvarez O. Importancia de la percepción de competencia y de la motivación en la salud mental de deportistas universitarios. Salud Pública Mex. 2010; 52(6): 517-523.

26. Muñoz-París MJ, Ruiz-Muñoz AM. Nivel de autoestima y correlación con comportamientos de riesgo en alumnos de la Universidad de Almería. Enferm Clin. 2008; 18(2): 70-6.

27. Ramos Bermúdez S, Alzate Salazar DA, Ayala Zuluaga JE, Franco Jiménez AM, Sánchez Valencia JA. Perfil de Fitness de los estudiantes de la Universidad de Caldas. Hacia promoc. Salud. 2009; 14(1): 23-34.

28. Páez Cala ML, Castaño Castrillón JJ. Estilos de vida y salud en estudiantes de una Facultad de Psicología. Psicol. caribe [Internet]. 2010 [citado 22 agosto 2012]
(25): 155-178. Disponible en: http://redalyc.uaemex.mx

29. Oviedo G, Morón de Salim A, Santos I, Sequera S., Soufrontt G, Suárez P. et al. Factores de riesgo de enfermedades crónicas no transmisibles en estudiantes de la carrera de Medicina: Universidad de Carabobo, Venezuela, 2006. Nutr Hosp. 2008; 23(3): 288-293.

30. Montero Bravo A, Úbeda Martín A, García González A. Evaluación de los hábitos alimentarios de una población de estudiantes universitarios en relación con sus conocimientos nutricionales. Nutr Hosp. 2006; 21(4): 466-73.

31. Sanabria-Ferrand P, González L, Urrego D. Estilos de Vida Saludable en Profesionales de la Salud Colombianos: Estudio Exploratorio. Rev fac med [Internet]. 2007 julio [citado 1 agosto 2012]; 15(2): 207-217. Disponible en: http://www.scielo.org.co 